\title{
Improved Decoupling for Low Frequency MRI Arrays using Non-conventional Preamplifier Impedance
}

Sanchez, Juan Diego; Johansen, Daniel Højrup; Hansen, Rie Beck; Hansen, Esben S. Szocska; Laustsen, Christoffer; Zhurbenko, Vitaliy; Ardenkjær-Larsen, Jan Henrik

Published in:

I E E E Transactions on Biomedical Engineering

Link to article, DOI:

10.1109/TBME.2018.2881203

Publication date:

2019

Document Version

Peer reviewed version

Link back to DTU Orbit

Citation (APA):

Sanchez, J. D., Johansen, D. H., Hansen, R. B., Hansen, E. S. S., Laustsen, C., Zhurbenko, V., \& ArdenkjærLarsen, J. H. (2019). Improved Decoupling for Low Frequency MRI Arrays using Non-conventional Preamplifier Impedance. I E E E Transactions on Biomedical Engineering, 66(7), 1940 - 1948.

https://doi.org/10.1109/TBME.2018.2881203

\section{General rights}

Copyright and moral rights for the publications made accessible in the public portal are retained by the authors and/or other copyright owners and it is a condition of accessing publications that users recognise and abide by the legal requirements associated with these rights.

- Users may download and print one copy of any publication from the public portal for the purpose of private study or research.

- You may not further distribute the material or use it for any profit-making activity or commercial gain

- You may freely distribute the URL identifying the publication in the public portal 


\title{
Improved Decoupling for Low Frequency MRI Arrays using Non-conventional Preamplifier Impedance
}

\author{
Juan D. Sánchez-Heredia, Daniel H. Johansen, Rie B. Hansen, Esben S. Szocska Hansen, Christoffer \\ Laustsen, Vitaliy Zhurbenko and Jan H. Ardenkjær-Larsen
}

\begin{abstract}
Objective: In this study, we describe a method to improve preamplifier decoupling in low frequency MRI receive coil arrays, where sample loading is low and coils exhibit a high $Q-$ factor. Methods: The method relies on the higher decoupling obtained when coils are matched to an impedance higher than 50 $\Omega$. Preamplifiers with inductive (and low resistive) input impedance, increase even further the effectiveness of the method. Results: We show that for poorly sample loaded coils, coupling to other elements in an array is a major source of SNR degradation due to a reduction of the coil Q-factor. An 8-channel ${ }^{13} \mathrm{C}$ array at $32 \mathrm{MHz}$ for imaging of the human head has been designed following this strategy. The improved decoupling even allowed constructing the array without overlapping of neighboring coils. Parallel imaging performance is also evaluated demonstrating a better spatial encoding of the array due to its non-overlapped geometry. Conclusion: The proposed design strategy for coil arrays is beneficial for low frequency coils where the coil thermal noise is dominant. The method has been demonstrated on an 8channel array for the human head for ${ }^{13} \mathrm{C}$ MRI at $3 \mathrm{~T}(32 \mathrm{MHz})$, with almost 2-fold SNR enhancement when compared to a traditional array of similar size and number of elements. Significance: The proposed method is of relevance for low frequency arrays, where sample loading is low, and noise correlation is high due to insufficient coil decoupling.
\end{abstract}

Index Terms- RF coil; SNR; ${ }^{13}$ C MRI; Hyperpolarization,

\section{INTRODUCTION}

$\mathrm{D}$ ISSOLUTION Dynamic Nuclear Polarization (dDNP) provides the means to increase the liquid state NMR signal-to-noise ratio (SNR) more than 10,000 fold [1] and has proven to be a powerful technique for the study of metabolism with ${ }^{13} \mathrm{C}$-enriched bioprobes in cancer and heart disease [2]-[4] . The method opens up new prospects for early detection of disease, disease progression and staging, as well as therapy monitoring. However, despite the large signal enhancement, the signal is weak and decays fast due to the low concentration of metabolic products and relaxation. Therefore, any improvement that can be harvested from e.g. MRI hardware and pulse sequences, will have tremendous impact on the quality of images.

In this study we demonstrate a method to obtain higher preamplifier decoupling by using non-conventional impedances for the coil matching and/or the input impedance of the preamplifier. More precisely, we show that matching coils to higher impedance than the noise-optimum of the preamplifier (often $50 \Omega$ ) can be beneficial to improve the decoupling when using purely resistive low input impedance preamplifiers. If we do not require the preamplifier impedance to be purely resistive, the decoupling can be improved further if the preamplifier has an inductive input impedance (with low real part).

The method is demonstrated by decoupling the elements of an 8 -channel ${ }^{13} \mathrm{C}$ head-coil $(32 \mathrm{MHz}$ at $3 \mathrm{~T}$ ) array. The coils were not overlapped with the purpose of improving the parallel imaging performance of the array. The realization is based on self-developed preamplifiers with input impedance $Z_{\mathrm{PRE}}=$ $(0.5+\mathrm{j} 67 \Omega)$ [5].

We show that if the achieved preamplifier decoupling is high enough, then each array element will have an impedance similar to the impedance of a single element in isolation, which is beneficial in terms of individual SNR of each element. Previous studies have shown that even in a high coupling situation, the combined SNR of the array is not degraded if full knowledge of the complex coupling coefficients can be obtained to calculate the optimal weights for the combination [6]-[8]. However, in the more general case where the weighting factors are not known a-priori, the method proposed here facilitates improved noise matching of each coil element, and also the subsequent image combination.

It should be mentioned, that non-overlapped arrays for ${ }^{1} \mathrm{H}$ imaging have been demonstrated before in [9]-[11]. Direct scaling these designs to lower frequencies is a tough challenge, and requires special attention to the thermal noise of the circuit used to tune and match the coils due to weaker sample loading at the ${ }^{13} \mathrm{C}$ frequency.
Manuscript received January 31, 2018. This work was supported in part by the Danish Council for Independent Research (DFF - 4005-00531) and the Danish National Research Foundation (DNRF124).

J. D. Sanchez-Heredia, D. H. Johansen, R. B. Hansen, V. Zhurbenko and J. H. Ardenkjær-Larsen are with the Center for Hyperpolarization in Magnetic
Resonance, Technical University of Denmark (DTU), 2800 Kgs. Lyngby, Denmark. J. H. Ardenkjær-Larsen is also with GE Healthcare, Denmark (correspondence e-mail: jhar@elektro.dtu.dk).

E. S. Szocska, Hansen and C. Laustsen are with the MR Research Centre, Department of Clinical Medicine, Aarhus University, Aarhus.

Copyright (c) 2018 IEEE. Personal use of this material is permitted. However, permission to use this material for any other purposes must be obtained from the IEEE by sending an email to pubs-permissions@ieee.org. 


\section{THEORY}

\section{A. Motivation}

Preamplifier decoupling has been the enabling technique that boosted the use of high density coil arrays in MRI. However, there are some limits for its use in the original version proposed by Roemer in [12], due to the impractical circuit values needed in some cases. The low gyromagnetic ratio of ${ }^{13} \mathrm{C}$ (four times lower than ${ }^{1} \mathrm{H}$ ), makes the design of ${ }^{13} \mathrm{C}$ coil arrays challenging due to the low sample loading (high Q), strong coupling between elements, and the difficulty of decoupling them without decreasing the SNR of the individual coils. Preamplifier decoupling as described in [12] (and further developed in [13]) can potentially overcome the problem, but for high-Q coils, a very low input impedance amplifier is needed in order to obtain a reasonable decoupling. However, for the case of ${ }^{13} \mathrm{C}$ at $3 \mathrm{~T}(32 \mathrm{MHz})$, commercially available preamplifiers have input impedance of more than $2.5 \Omega$, which for high-Q coils $(>300)$ provide very modest decoupling $(<13$ $\mathrm{dB}$ ) in the standard configuration. Therefore, at low frequencies, these two effects add up: low sample loading of the coil (and therefore high-Q), and relatively high input impedance of the preamplifier.

It can be shown that for high-Q coils, a series tuned/parallel matched scheme introduces significantly more noise due to the high current flow through the matching capacitor (due to the parasitic Equivalent Series Resistance (ESR)). Instead, a parallel tuned/series matched scheme should be preferred, as the current flow through the matching network is minimal and thus the noise contribution [14].

Preamplifier decoupling proposed in [13] consists of a pi network for matching the coil and exploits the additional degree of freedom given by the extra capacitor added to the network in order to create the preamplifier decoupling condition. This circuit has the advantage that the value of one component can be arbitrarily chosen, meaning that the coil designer can adjust the component values of the whole circuit in order to achieve practical capacitances and inductances. Furthermore, the coil designer can use this degree of freedom to implement the matching network in a way that resembles a parallel-tuned series-matched circuit, with the consequent improvement of the noise performance. This is, most likely, the explanation of the $10 \%$ increase of the SNR that Reykowski and colleagues found in [13] compared to the traditional circuit though the number of components in the total circuit is higher.

\section{B. Improved Decoupling using Non-Conventional Matching and Preamplifier Impedance}

The circuit in Figure 1 allows preamplifier decoupling using traditional (purely resistive) low input impedance preamplifiers. In this circuit the inductor $\mathrm{L}_{4}$ has a strong role on both the matching of the coil to the reference impedance for noise matching $\left(\mathrm{Z}_{0}\right)$ and the preamplifier decoupling creating a circular dependence of the two conditions [13].

In order to get a simpler analytical solution we consider the case where the inductor $\mathrm{L}_{4}$ can be integrated as part of the preamplifier design, while keeping the optimal noise impedance as $Z_{0}$. In that case the preamplifier will show an inductive impedance, which does not contribute to the coil matching to $Z_{0}$. Therefore, the matching condition of the coil is given by $Z_{\mathrm{IN}}$ ' (as depicted in Figure 1) equal to $Z_{0}$. For an arbitrary value of $X$, one can demonstrate (see Appendix A) that this condition leads to (1) and (2).

$$
\begin{gathered}
\mathrm{X}_{\mathrm{T}}=\beta \cdot \frac{\mathrm{X}_{\mathrm{L} \pm} \sqrt{\frac{\mathrm{R}_{\mathrm{C}}{ }^{3}-\mathrm{R}_{\mathrm{C}}{ }^{2}+\frac{\mathrm{R}_{\mathrm{C}} \cdot \mathrm{X}_{\mathrm{L}}{ }^{2}}{\beta}}{\beta-R_{C}}} ; \quad \beta=\frac{\mathrm{Z}_{0} \cdot \mathrm{X}^{2}}{\mathrm{Z}_{0}{ }^{2}+\mathrm{X}^{2}}}{\mathrm{X}_{\mathrm{M}}=\left[\frac{-\mathrm{X}_{\mathrm{T}} \cdot\left(\mathrm{R}_{\mathrm{C}}{ }^{2}+\mathrm{X}_{\mathrm{L}} \cdot\left(\mathrm{X}_{\mathrm{L}}-\mathrm{X}_{\mathrm{T}}\right)\right.}{\mathrm{R}_{\mathrm{C}}{ }^{2}+\left(\mathrm{X}_{\mathrm{L}}-\mathrm{X}_{\mathrm{T}}\right)^{2}}\right]-\left[\frac{\mathrm{X} \cdot \mathrm{Z}_{0}{ }^{2}}{\mathrm{Z}_{0}{ }^{2}+\mathrm{X}^{2}}\right]}
\end{gathered}
$$

What we have, so far, is a parallel-tuned series-matched coil where the extra capacitor $\mathrm{X}$ provides a degree of freedom, such that one can choose between an infinite number of solutions for the values of capacitors $X_{T}, X_{M}$ and $X$.

If we assume that the impedance of the inductor $\mathrm{X}_{\mathrm{L} 4}$ is part of the preamplifier, we can calculate the value of $Z_{\mathrm{PRE}}$ ' needed to obtain preamplifier decoupling (see Appendix A). This can be done by maximizing the impedance seen at the terminals of the loop ( $\left.\mathrm{Z}_{\text {MATCH }}\right)$, which leads to (3).

$$
\mathrm{Z}_{\mathrm{PRE}}{ }^{\prime}=\mathrm{j} \cdot \frac{\mathrm{X} \cdot\left(\mathrm{X}_{\mathrm{T}}+\mathrm{X}_{\mathrm{M}}\right)}{\left(\mathrm{X}+\mathrm{X}_{\mathrm{T}}+\mathrm{X}_{\mathrm{M}}\right)}
$$

Where, for a given coil $\left(\mathrm{X}_{\mathrm{L}}, \mathrm{R}_{\mathrm{C}}\right)$ and an arbitrary value of $\mathrm{X}$, we can obtain the required values of $X_{T}, X_{M}$ and $Z_{P R E}$ ' to tune and match the coil to $Z_{0}$ and create preamplifier decoupling. $Z_{\mathrm{PRE}}$ ' will be positive (inductive) when $\mathrm{X}_{\mathrm{T}}, \mathrm{X}_{\mathrm{M}}$ and $\mathrm{X}$ are capacitive.

An estimation of the additional decoupling given by a preamplifier with input impedance $Z_{\mathrm{PRE}}$ ' can be calculated as the difference in current through the loop when matched and connected to a $50 \Omega$ load, and the case where the preamplifier is connected. For a given induced voltage at the loop terminals, the current flow will be inversely proportional to the impedance shown to the loop by the matching network $\left(\mathrm{Z}_{\mathrm{MATCH}}\right)$ when the preamplifier decoupling condition is satisfied (4).

$$
\mathrm{Z}_{\mathrm{MATCH}}=\frac{\mathrm{X}_{\mathrm{T}}^{2} \cdot \mathrm{X}^{2}}{\operatorname{Re}\left\{\mathrm{Z}_{\mathrm{PRE}}\right\} \cdot\left[\mathrm{X}+\mathrm{X}_{\mathrm{T}}+\mathrm{X}_{\mathrm{M}}\right]^{2}}
$$

A consequence of (4) is that the decoupling obtained for a given preamplifier impedance is inversely proportional to the term $\left[\mathrm{X}+\mathrm{X}_{\mathrm{T}}+\mathrm{X}_{\mathrm{M}}\right]$. For a high- $\mathrm{Q}$ coil, the matching impedance $\mathrm{X}_{\mathrm{M}}$ tends to infinite, and therefore dominates the whole term. Therefore, lowering that impedance increases the decoupling (as it increases the blocking impedance $\mathrm{Z}_{\text {МАTCH}}$ ). Looking at the

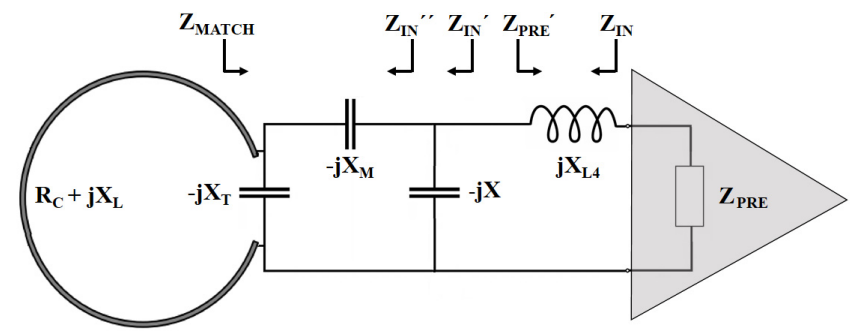

Fig. 1. Preamplifier decoupling matching network, as described in [13]. 
equations that describe the circuit in Figure 1 [13], there are two ways to decrease $\mathrm{X}_{\mathrm{M}}$ : one is to choose a higher reference impedance $\mathrm{Z}_{0}$; the second one, is to reduce $\mathrm{X}_{\mathrm{L} 4}$, while keeping the rest of the circuit unmodified. The second option requires that the preamplifier has an inductive impedance component, in order to keep the preamplifier decoupling condition for a given value of the impedance $\mathrm{X}$.

\section{SNR Reduction due to Mutual Coupling}

Mutual coupling between the coil elements in an array will significantly reduce the unloaded Q-factor of every individual coil. This effect can often be disregarded in arrays with high sample loading, where the loaded Q is low, and the main Q reduction comes from the currents induced in the sample. However, if sample loading is low (low frequencies and/or small elements), there will be a significant reduction of the individual $\mathrm{Q}$ factor of each element due to the induced currents in other elements of the array.

Having coil elements with lower Q-factors has an immediate effect on the SNR of the MRI experiment, since the induced voltage at each coil will be lower, while the noise floor (for a given matching) will remain the same. Therefore, lower Qfactor, means finally lower SNR. In Section IV.B we show this effect experimentally.

One could indeed allow coupling between the array elements, and tune the coil array in such a way that the whole array works electromagnetically as one resonant structure. However, for arrays with many elements, the degree of complexity of the tuning process of such a structure can be very high.

The method proposed here pursues the opposite approach: If the decoupling between coil elements is high enough, the Qfactor of each coil will be the same in free space than when the element is placed in the array. This simplifies the tune and matching of the array, since one could simply tune and match every individual element separately and then place it in the array without the need of re-tuning and re-matching.

\section{METHODS}

\section{A. Preamplifier Decoupling}

In order to validate the analysis in the previous section, a simple 2-channel array was built, using wire loop coils (coil diameter $50 \mathrm{~mm}$, wire diameter $2.3 \mathrm{~mm}$ ) separated $75 \mathrm{~mm}$ center to center. Experiments were repeated using a commercial preamplifier with resistive low input impedance (WMA32C, WanTCom, Chanhassen, MN, USA), with the coils matched to $50 \Omega$ and $100 \Omega$, and a self-developed preamplifier [5] with an input impedance $Z_{\mathrm{PRE}}=(0.5+\mathrm{j} 67 \Omega)$ and the coils matched to $50 \Omega$. The parameters of the preamplifiers are shown in Table 1. These three combinations of reference and preamplifier impedances are referred through the rest of the paper as Configurations 1, 2 and 3.

The Noise Figure (NF) of the preamplifiers was measured for the cases where $Z_{0}$ is $50 \Omega$ (Configurations 1 and 3 ) using a spectrum analyzer with noise measurement capability (PSA
E4440A, Keysight, CA, USA). The NF for Configuration 2, where $Z_{0}$ is $100 \Omega$, was obtained from the manufacturer.

The preamplifier decoupling performance was calculated from the increase of impedance shown to the coil by the matching network ( $\left.\mathrm{Z}_{\mathrm{MATCH}}\right)$ between the $50 \Omega$ and the preamplifier loads. The Q-factor of the coils when mounted in the array was also measured from the reflection coefficient $\left(S_{11}\right)$ when the other coil was terminated with the preamplifier, using the following relation:

$$
Q=\frac{f_{0}}{\Delta B W_{45 \mathrm{deg}}}
$$

where $\Delta B W_{45} \mathrm{deg}$ is the bandwidth defined by the \pm 45 degree phase points of the $\mathrm{S}_{11}$ response [15].

\section{B. SNR Measurements}

In order to quantify the impact that better decoupling can have on the final SNR of an MRI experiment, SNR measurements were carried out using the 2-element array described before.

The measurements were done inducing a signal on the array using a pickup loop [16] and measuring the SNR for the different preamplifier configurations. The coils were placed inside a shielded box (R\&S CMW-Z10, Munich, Germany), a signal of $-90 \mathrm{dBm}$ was fed to the pickup loop using a signal generator. The setup is shown in Figure 2.

Preamplifiers were connected to the 2 coils, with the three configurations described before, and the spectrum at the output of Coil 1 was recorded with the same spectrum analyzer used for NF measurements. The SNR of this measurement was then compared to the SNR provided by the same coil, when Coil 2 was not present. The coils were tune and match at each configuration, to ensure that the noise matching of the preamplifier is the one shown in Table 1 for each configuration. This comparison allows us to quantify the SNR reduction on one coil due to the presence of the other one.

\section{A non-overlapped 8-Channel Head Coil}

An advantage of higher preamplifier decoupling is that one can consider not using decoupling through coil overlap and therefore improve the spatial encoding of the coil array for

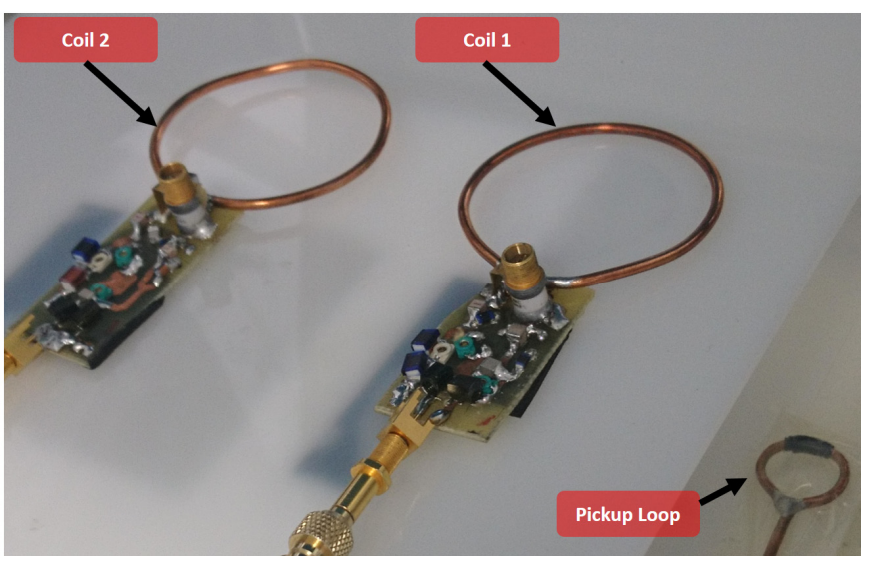

Fig. 2. Setup used for SNR measurements: two $50 \mathrm{~mm}$ coils separated $75 \mathrm{~mm}$ center-to-center, with a small pickup loop placed close to one of the coils, and used to induce a signal. 
TABLE I

DiFFERENT CONFIGURATIONS OF PREAMPLIFIER AND MATCHING IMPEDANCE

\begin{tabular}{|c|c|c|c|c|c|c|c|}
\hline Configuration & $\mathrm{Z}_{\mathrm{PRE}}[\Omega]$ & $\overline{Z_{0}[\Omega]}$ & $\mathrm{NF}[\mathrm{dB}]$ & $\overline{\mathrm{C}_{\mathrm{T}}[\mathrm{pF}]}$ & $\mathrm{C}_{\mathrm{M}}[\mathrm{pF}]$ & $\overline{\mathrm{C}_{\mathrm{X}}[\mathrm{pF}]}$ & $\overline{\mathrm{L}_{4}[\mathrm{nH}]}$ \\
\hline 1 & $2.5+\mathrm{j} 0$ & 50 & 0.55 & 239 & 6.5 & 75.6 & 300 \\
\hline 2 & $2.5+\mathrm{j} 0$ & 100 & 0.82 & 237 & 9.1 & 73 & 300 \\
\hline 3 & $0.5+j 67$ & 50 & 0.76 & 236 & 9.7 & 64.6 & 0 \\
\hline
\end{tabular}

$\mathrm{C}_{\mathrm{T}}, \mathrm{C}_{\mathrm{M}}, \mathrm{C}_{\mathrm{X}}$ and $\mathrm{L}_{4}$, are the lumped element values associated to the impedances $\mathrm{X}_{\mathrm{T}}, \mathrm{X}_{\mathrm{M}}, \mathrm{X}$ and $\mathrm{X}_{\mathrm{L} 4}$.

parallel imaging. Parallel imaging is particularly advantageous for hyperpolarized spectroscopic imaging, where the magnetization is not recoverable, and (ideally) there is no SNR penalty associated with the acceleration ([17], [18]).

Using the best preamplifier decoupling configuration described in the previous section (Configuration 3), a nonoverlapped 8-channel coil array for ${ }^{13} \mathrm{C}$ imaging of the human head at $3 \mathrm{~T}$ was designed. The number of elements was limited to eight, since that was the maximum number of ${ }^{13} \mathrm{C}$ receive channels allowed by our imaging setup. A cylinder, open in both ends, (252 mm ID, $254 \mathrm{~mm}$ OD) made of fiberglass (Elektro Isola, Denmark) was used as coil holder, and the coils and associated electronics were placed on the outer part of the cylinder, keeping the inner part clear for easy access of the sample. The $80 \mathrm{~mm}$ diameter circular loops, were made from copper wire with a diameter of $2.3 \mathrm{~mm}$, and attached to the fiberglass holder with cable strips with $18 \mathrm{~mm}$ separation between nearest neighbors.

Ceramic chip capacitors (Johanson Technology, Camarillo, CA, USA) were used where fixed capacitances were needed, high-Q trimmers (Johanson Technology, Camarillo, CA, USA) where added in parallel to the tuning capacitors for fine tuning, while slightly lower Q trimmers (Sprague Goodman, Westbury, NY, USA) were used on the rest of the circuit for matching and fine-tuning of the preamplifier decoupling. Surface mount inductors were used for the active decoupling circuit (Coilcraft, Cary, IL, USA), which was completed with PIN diodes (MA4P7470F-1072T, MACOM, Lowell, MA, USA). Fast switching diodes (UMX9989AP, Microsemi, Aliso Viejo, CA, USA) were used for the passive decoupling and preamplifier input protection. All the described components have nonmagnetic terminations.

The array was simulated in CST (Darmstadt, Germany), with the coils modelled using wire with a cross section of $4.16 \mathrm{~mm}^{2}$ and square profile. A female head model (Ella, Virtual Family [19]) was used to emulate sample loading. Using the circuit simulation feature of CST, the coil arrays were tuned and matched, including losses in the lumped elements. The preamplifier decoupling performance was included in the simulation, in order to have a realistic estimation of the decoupling between elements. This was done by terminating the port of each coil element with the impedance shown at the input of the preamplifier $\left(Z_{\mathrm{PRE}}=0.5+\mathrm{j} 67 \Omega, Z_{0}=50 \Omega\right)$. The $\mathrm{B}_{1}{ }^{-}$ field component (normalized to $1 \mathrm{~W}$ of accepted power) was then calculated, as well as the SENSE g-factor for an acceleration rate $(\mathrm{R})$ of 4 [20].

For a loop diameter of $80 \mathrm{~mm}$, the question arises of whether capacitive segmentation is needed in order to avoid additional noise from the electric field across the loop. A rule of thumb is to segment the coil (at least) every $\lambda / 20$ [21], $\lambda$ being the wavelength at $32 \mathrm{MHz}(9.4 \mathrm{~m}$ in free space corresponding to segments of $47 \mathrm{~cm}$ ). The perimeter of an $80 \mathrm{~mm}$ diameter circular loop is $25 \mathrm{~cm}$ and, in principle, it would not require segmentation. This hypothesis was confirmed experimentally, by comparing two $80 \mathrm{~mm}$ coils, one segmented and one unsegmented with and without loading (see Appendix B). While, for this particular coil size and frequency, both segmented and non-segmented coils behave very similarly in terms of losses, using non-segmented coils also allows us to illustrate the effectiveness of the proposed matching network and decoupling strategy for arrays with even smaller loop size (or lower frequency), where the benefits of non-segmented loops are even more significant [14].

\section{Coil Matching Network}

In order to detune the receiving coils during transmission [22], [23], a modification of the circuit proposed in [13] is used, in combination with the active decoupling scheme proposed in [14] (Figure 3). This circuit allows keeping the active decoupling independent of the inductance used for the preamplifier decoupling, which is practical when the preamplifier decoupling needs to be fine-tuned (as is the case in high Q coils). Additionally, a secondary (passive) decoupling trap was added, in accordance with [24]. Due to the lack of segmenting capacitors in our design, this trap was implemented using part of the detecting loop, in parallel with a capacitor activated through crossed diodes.

The advantages of this circuit are several:

- Splitting the matching capacitors $\left(\mathrm{C}_{\mathrm{M}}\right)$ allows introduction of the active decoupling circuitry without having DC voltage on the detecting loop.

- The active decoupling circuitry is tuned to the desired frequency with the inductors $\mathrm{L}_{1}$ and $\mathrm{L}_{2}$, and is independent of the preamplifier impedance $Z_{\text {PRE. }}$ This

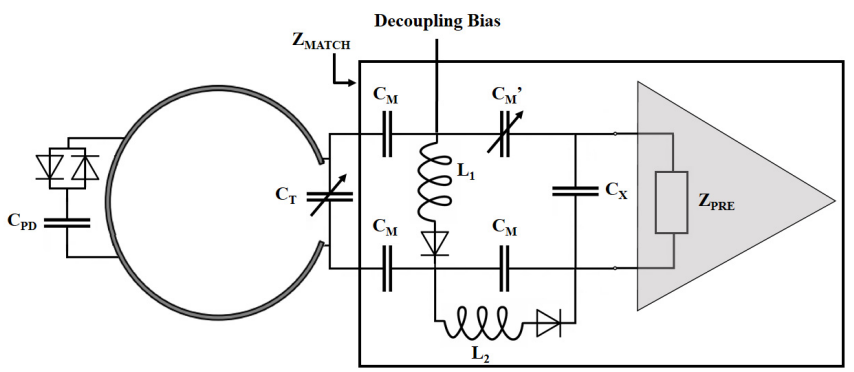

Fig. 3. Circuit used to tune and match the coils of the 8-channel array. Adding variable capacitors in parallel to the capacitances $\mathrm{C}_{\mathrm{T}}, \mathrm{C}_{\mathrm{M}}$ ' and $\mathrm{C}_{\mathrm{X}}$ allows tuning independently the resonance frequency of the coil, the matching and the preamplifier decoupling, respectively. The active decoupling from the transmitter is tuned with the inductors $\mathrm{L}_{1}$ and $\mathrm{L}_{2}$. The values used for the lumped elements are: $\mathrm{C}_{\mathrm{T}} \approx 120 \mathrm{pF}, \mathrm{C}_{\mathrm{M}}=47 \mathrm{pF}, \mathrm{C}_{\mathrm{M}}{ }^{\prime}=28-50 \mathrm{pF}, \mathrm{L}_{1}=1000 \mathrm{nH}$, $\mathrm{L}_{2}=520 \mathrm{nH}$ and $\mathrm{C}_{\mathrm{X}}=38-60 \mathrm{pF} . \mathrm{Z}_{\mathrm{MATCH}}$ is the equivalent impedance of the whole matching network. 
permits active decoupling even when the inductance used for preamplifier decoupling is not part of the active decoupling circuit (because it is part of the preamplifier).

- The preamplifier decoupling frequency can be adjusted by tuning of capacitor, $\mathrm{C}_{\mathrm{X}}$, which is easier in practice than using an inductor (as one would do using the circuit in [12]).

- By choosing a relatively low value of capacitance $C_{X}$, this matching network resembles a parallel-tuned series-matched circuit, which, for high-Q coils, has lower thermal noise than its series-tuned parallelmatched equivalent.

The preamplifiers used were built using similar capacitors and inductors, including in this case also nonmagnetic resistors (Passive Plus, Huntington, NY, USA). A pHeMT transistor (ATF 58143, Broadcom, San Jose, CA, USA) was used as active element for the preamplifier, and biased in a common source fashion as described in [5]. The fabricated coil array is shown in Figure 7.a.

The Q-factor of each coil was directly measured (by disconnecting its preamplifier, and measuring the $S_{11}$ with a VNA (E5062A, Keysight, Santa Rosa, CA, USA). This process was repeated with the coil unloaded, and sample loaded. The phantom used to test this coil is a cylinder with $250 \mathrm{~mm}$ of diameter and $200 \mathrm{~mm}$ long, filled with $\left({ }^{13} \mathrm{C}\right.$ natural abundance) ethylene glycol. The ethylene glycol used to fill the phantom has a $\varepsilon_{\mathrm{R}}=40$, and low conductivity $(\approx 0.1[\mathrm{~S} / \mathrm{m}])$. For this study, we decided not to increase the conductivity of this phantom in order to use it as an extreme case of poor loading for testing coils arrays. Since the main problems of coil arrays at low frequency arise from the high Q-factor that the coils maintain during operation, especially from the decoupling perspective, if a coil array can perform well with a phantom with low loading, it will also perform well with real samples.

Imaging experiments were performed at a clinical $3 \mathrm{~T}$ scanner (Signa HDx, GE Healthcare, Waukesha, WI, USA). The transmit coil is a $40 \mathrm{~cm}$ diameter volume coil of the clamshell type (GE Healthcare, Waukesha, WI, USA) [4][25].

Data were acquired using a Cartesian CSI sequence with a $24 \times 24$ matrix, FOV of $300 \times 300 \times 100 \mathrm{~mm}^{3}, 1$ average, flip angle of $90^{\circ}$ and a repetition time of $1 \mathrm{~s}$ resulting in a total scan time of $9 \mathrm{~min}$ and $36 \mathrm{~s}$. This dataset was used first to extract the sensitivity profiles of the coil array elements [26], and second to create three different retrospectively undersampled CSI datasets with $\mathrm{R}$ of 2,3 , and 4 , respectively. This was done to test the parallel imaging performance of the array. Retrospective undersampling was done by replacing lines in kspace with zeros, e.g. every second line for $R=2$. Each undersampled dataset was reconstructed using conventional SENSE [20] using the extracted coil profiles, and subsequently used for estimating the associated g-factor map.

The data acquisition and postprocessing were repeated with a commercial 8 channel array (GE Healthcare, Waukesha, WI, USA), formed by two paddles of 4 overlapped rectangular loops of $50 \times 100 \mathrm{~mm}$ [25] (Figure 7.d). This array is used as reference, since it is built using a more traditional design including decoupling via overlap, and preamplifier decoupling as originally described by Roemer et al. in [12]. This comparison to a state-of-the-art ${ }^{13} \mathrm{C}$ array (the best commercial ${ }^{13} \mathrm{C}$ coil array available in our lab), serve us to validate that we are not sacrificing the SNR performance of the array by the use of non-overlapped elements.

The phantom used to emulate sample loading is the same cylindrical phantom described in the previous section. The results were also compared to the numerical simulation.

\section{RESULTS}

\section{A. Preamplifier Decoupling}

The obtained decoupling for each configuration is shown in Figure 4.a., calculated from the formulation given in Annex A. The measured $\mathrm{S}_{11}$ and coil Q-factor are also shown in Figure 4.b, for the three different preamplifier configurations and compared to the original Q-factor of one coil in isolation. The obtained Q-factor is consistent with the obtained decoupling.

\section{B. SNR Loss Due to Coupling}

The SNR results measured on the 2-channel array described before are shown in Figure 5 for the three preamplifier configurations, and compared to the same measurement with only one coil (left column). The measured SNR is very similar for the three configurations when only one coil is present, but it gets lower when the second coil is added. The reduction is especially significant for Configuration 1, where SNR goes below $50 \%$ of the SNR measured with only one coil. Both Configurations 2 and 3 show a better performance, with Configuration 3 showing the best SNR.

For these measurements the coils are re-tuned and rematched for every case, therefore ensuring that the impedance shown to the preamplifier is the same whether we measure with Coil 1 alone, or with Coil 2 present. That means that the noise levels are the same, and the SNR difference comes from a higher signal intensity when the decoupling is higher (and so it is the coil Q-factor). It's important to note that better SNR is obtained in configurations 2 and 3, despite the fact that the noise matching of the preamplifier is worse in those cases $(\mathrm{NF}=0.55$ $\mathrm{dB}$ for Configuration 1, $0.82 \mathrm{~dB}$ for Configuration 2 and 0.75 for Configuration 3). Therefore, the SNR improvement can only be attributed to a lower coil noise contribution of the coils. 

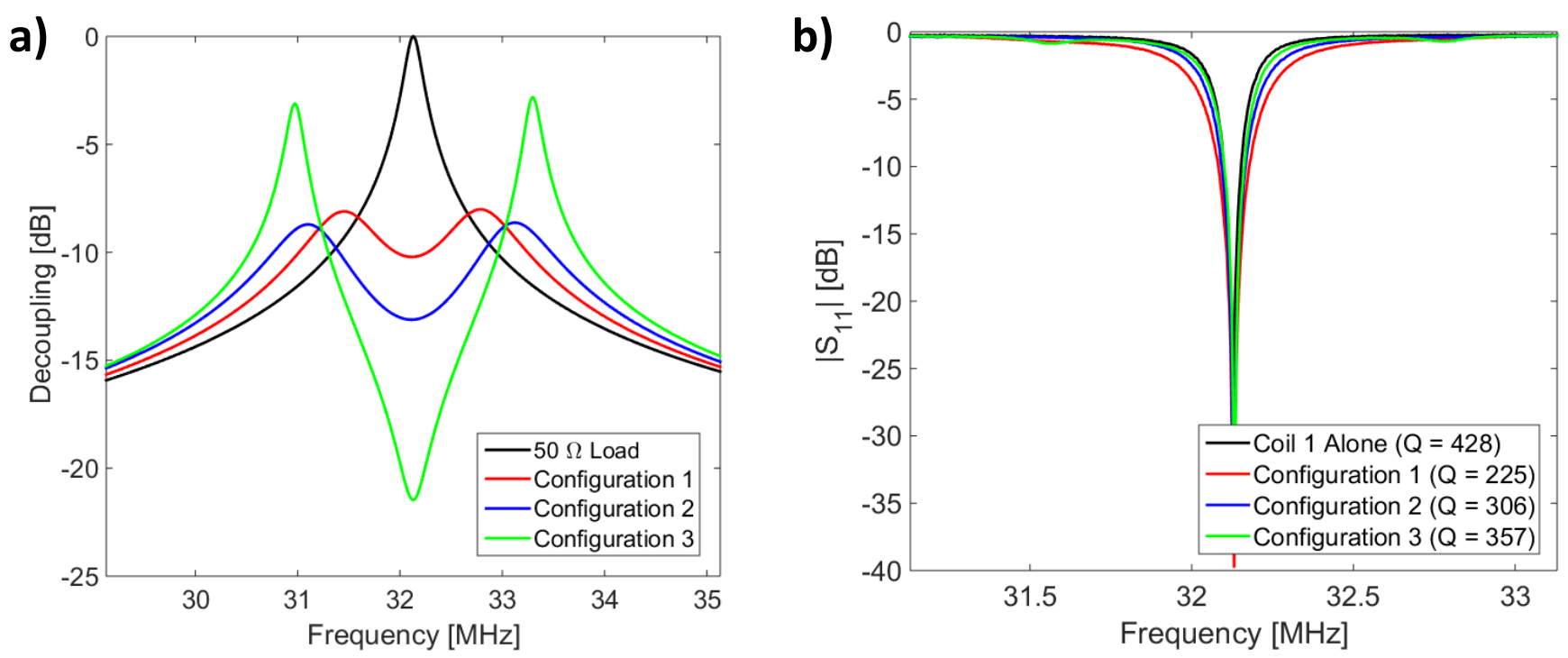

Fig. 4. a) Simulated decoupling for each of the preamplifier configurations, compared to the case where the coils are terminated in a $50 \Omega$ load (black line). b) Measured $\mathrm{S}_{11}$ of Coil 1 in Figure 2, when Coil 2 is terminated with its preamplifier in the three different configurations, compared to the case where Coil 1 is placed alone.

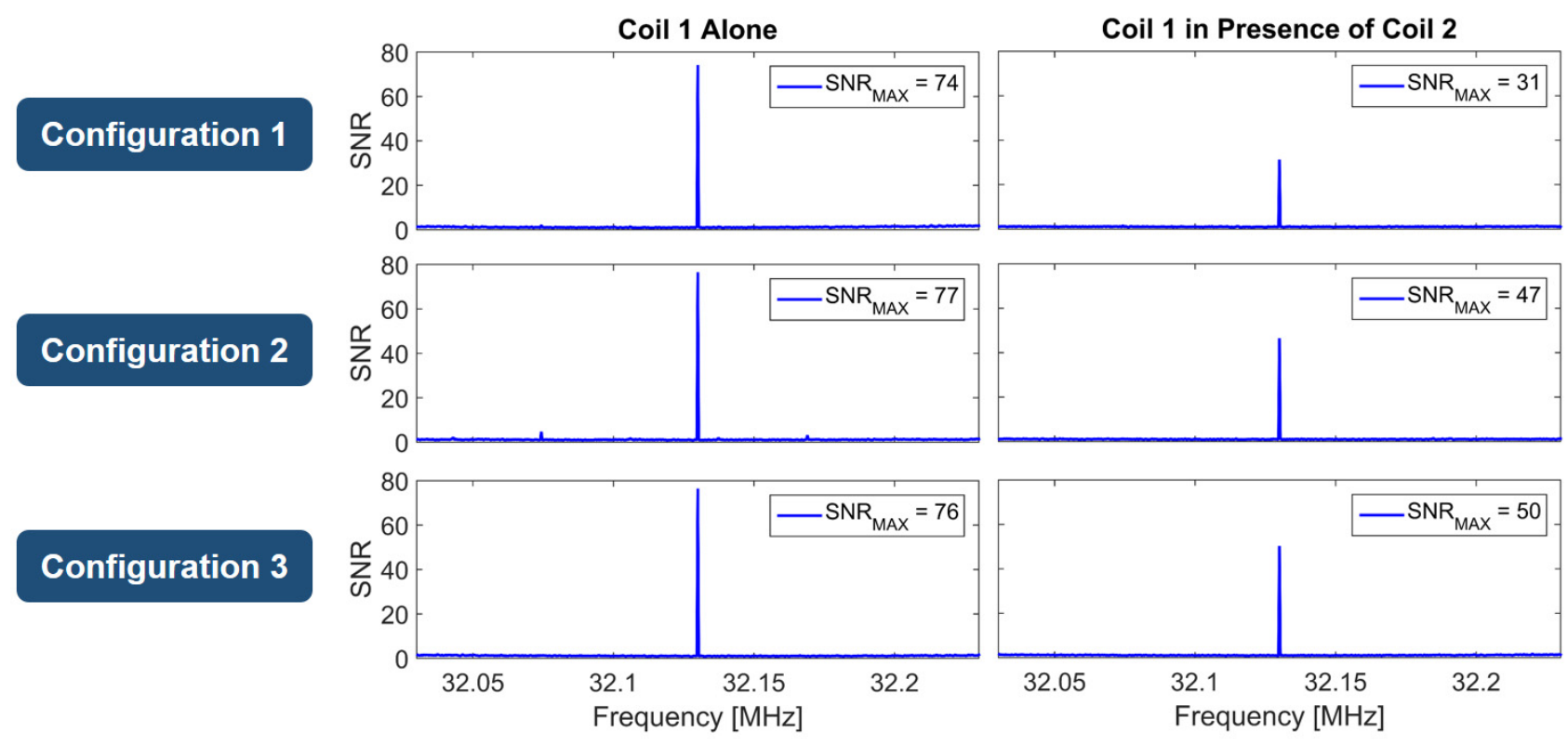

Fig. 5. SNR degradation of the signal detected by Coil 1 due to the presence of Coil 2, for the three different preamplifier configurations..

\section{Array Performance}

The simulated $\mathrm{B}_{1}^{-}$for the array is shown in Figure 6.b). The SENSE g-factor for an $\mathrm{R}$ of 4 , was also calculated [20], based on the individual sensitivity profiles of the simulation (Figure 6.c)). The calculated g-factor maps show the good parallel imaging performance of the array, with the worst-case voxel having a g-factor value of 1.41 .

The simulated and measured Q-factors of each coil element are shown in Table 2, for both unloaded and loaded cases. The results show good agreement between the numerical simulations and the bench characterization. Each coil element maintains a high Q-factor when mounted in the array (with the rest of coils present and tuned), with no split resonance due to coupling. The measured unloaded Q of the different elements is higher than 280, compared to approximately 420 for an isolated coil element.

The sum-of-squares combined image is shown in Figure 7.b) and compared to a similar one obtained with the commercial array (Figure 7.e)). From these two images, we see that the SNR provided by the fabricated array is higher at both the surface and the center of the phantom, which confirms the lower coil noise contribution in the fabricated array.

Figure 8 shows the SNR profiles of each individual coil element. While there are small differences between the performance of the different array elements due to the slightly different cross coupling between elements, the profiles are

Copyright (c) 2018 IEEE. Personal use of this material is permitted. However, permission to use this material for any other purposes must be obtained from the IEEE by sending an email to pubs-permissions@ieee.org. 


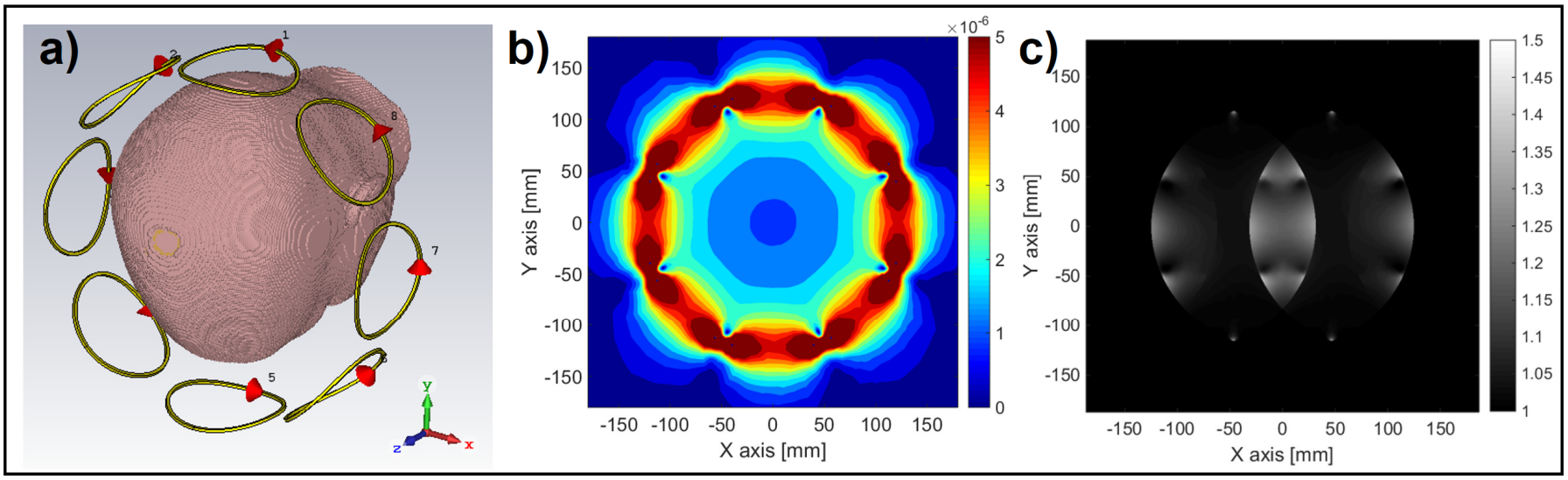

Fig. 6. a) Simulated array model, b) $\mathrm{B}_{1}^{-}[\mathrm{T}]$ per unit of accepted power and c) SENSE g-factor for an acceleration factor of 4, for the designed non-overlapped 8channel array.
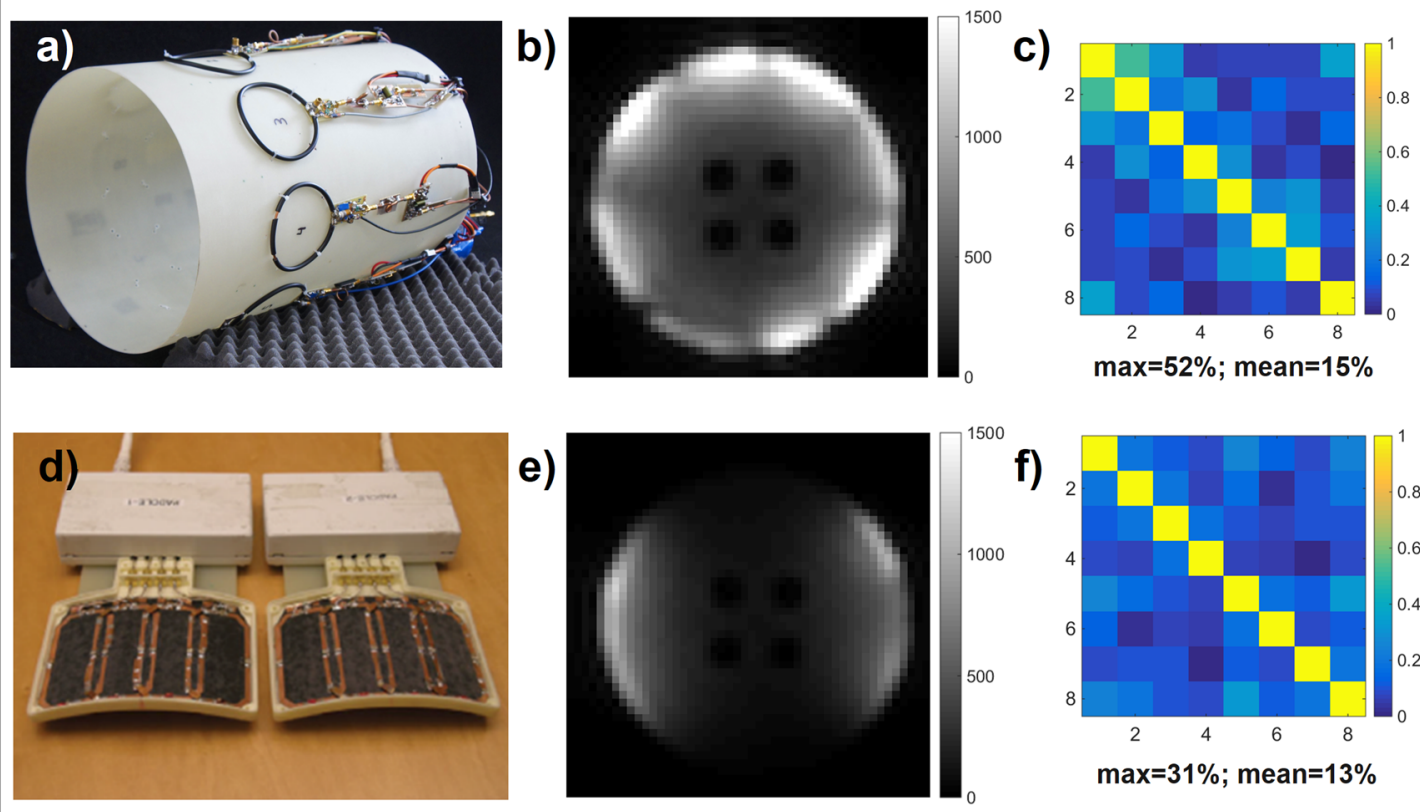

e)

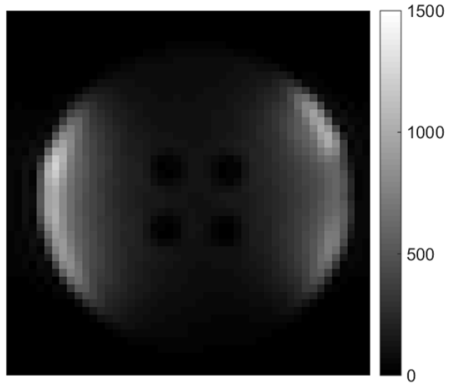

f)

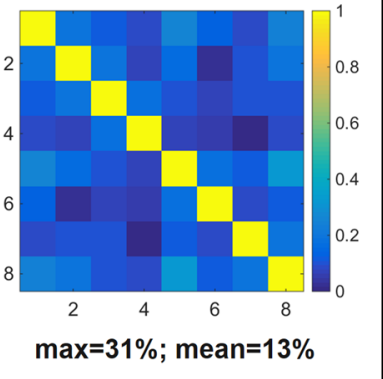

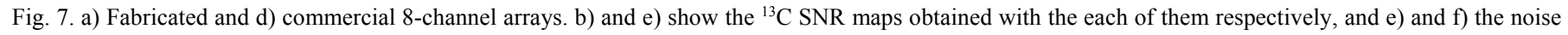
correlation matrices.

clearly distinguishable from each other, showing in general good decoupling and spatial encoding.

Figure 7.c) and f) show the measured noise correlation matrices for both coil arrays respectively. Again, we see some small differences between the correlation of individual pairs of coils, but the average correlation level is similar in both coil arrays ( $15 \%$ vs. $13 \%)$, despite of the non-overlapping geometry used in our array.

The estimated SENSE g-factor maps for three different acceleration rates $(2,3$, and 4$)$ are shown in Figure 9.a) for the fabricated array and b) for the commercial one. While both arrays have negligible g-factors for $\mathrm{R}=2$, the fabricated array has superior parallel imaging performance for $\mathrm{R}=3$ and $\mathrm{R}=4$ in terms of lower g-factors and hereby less noise amplification. The measured $\mathrm{g}$-factor distribution for an $\mathrm{R}=4$ is similar to the simulated map (Figure 6.c), however slightly higher in general. This could be explained by the extra coupling introduced by the transmit coil, which was not included in the simulation.
TABLE II

SimUlated AND MEASURED Q-FACTORS OF ALL THE COIL ELEMENTS WHEN MOUNTED ON THE ARRAY.

\begin{tabular}{l|cccc}
\hline \hline & \multicolumn{2}{|c}{ Numerical Simulations } & \multicolumn{2}{c}{ Bench Measurements } \\
& QUN $_{\text {UN }}$ & QLO & QUN & QLO \\
\hline Coil 1 & 303 & 194 & 306 & 285 \\
Coil 2 & 303 & 214 & 323 & 309 \\
Coil 3 & 303 & 235 & 316 & 295 \\
Coil 4 & 303 & 239 & 306 & 286 \\
Coil 5 & 303 & 240 & 306 & 295 \\
Coil 6 & 303 & 242 & 335 & 304 \\
Coil 7 & 303 & 238 & 296 & 278 \\
Coil 8 & 303 & 223 & 282 & 268 \\
\hline \hline
\end{tabular}

$\mathrm{Q}_{\mathrm{UN}}=$ non sample-loaded Q-factor, $\mathrm{Q}_{\mathrm{LO}}=$ sample-loaded Q-factor

\section{DisCUSSION}

We show in this work that mutual coupling between elements can be a major source of SNR degradation in low frequency coil arrays, where sample loading is low, and the Q-factor of the 
Coil 1

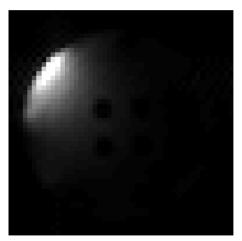

Coil 5

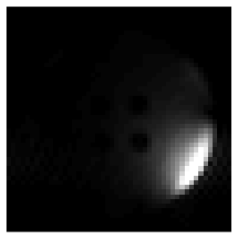

Coil 2

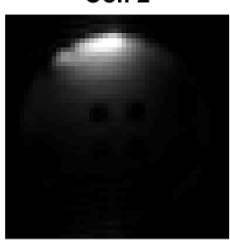

Coil 6

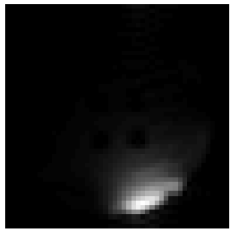

Coil 3

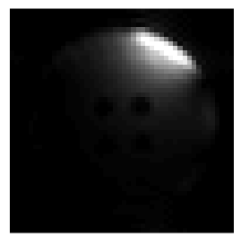

Coil 7

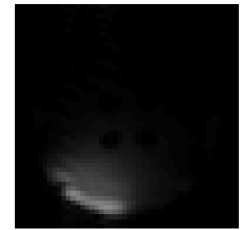

Coil 4

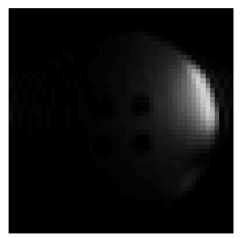

Coil 8

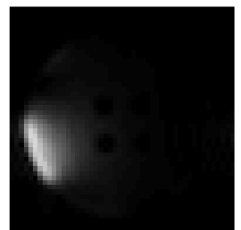

Fig. 8. Individual coil element images for the fabricated 8-channel head array, using a phantom filled with ethylene glycol (with natural abundance of ${ }^{13} \mathrm{C}$ ).

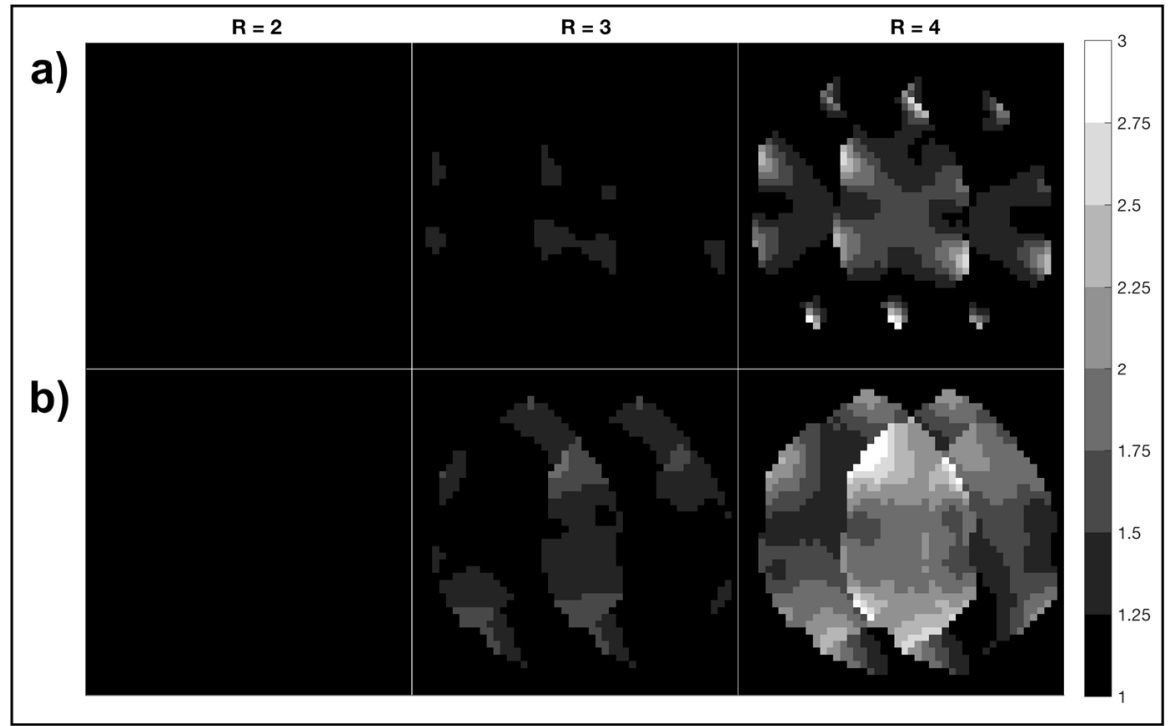

Fig. 9. Measured SENSE g-factor maps for three different acceleration rates (2, 3 and 4). a) Fabricated 8-channel head coil array, and b) commercial 8-channel paddle coil array.

coils can be heavily reduced by induced currents in other elements of the array. To overcome this problem, nonconventional impedances (different from $50 \Omega$ ) can be used for coil matching to obtain better preamplifier decoupling when using the circuit proposed in [13]. In this study, we show that matching to a higher impedance using traditional low input (real) impedance preamplifiers improves the preamplifier decoupling, and can be beneficial in terms of SNR for coils where the coil noise contribution is dominant. This effect can be exploited even further, if the preamplifier presents an inductive input impedance, therefore allowing the matching to a given reference impedance $Z_{0}$ with a higher capacitance value ( $\mathrm{X}_{\mathrm{M}}$ in Figure 1).

This new decoupling method has been successfully implemented in an 8-channel head coil array for ${ }^{13} \mathrm{C}$ at $3 \mathrm{~T}$ (32 $\mathrm{MHz}$ ). The improved decoupling, even allows to refrain from using overlap decoupling between neighboring coils, with the consequent simplification of coil array fabrication and improved spatial encoding of the array. A modification of the matching network proposed in [13] is also proposed, in order to allow active decoupling, passive decoupling and separate tuning of the preamplifier decoupling. These modifications, besides of practical, also allow minimizing the noise contribution of the lumped elements in the matching network. Indeed, an average $80 \%$ SNR enhancement has been obtained when comparing to a commercial array of similar size and number of elements. The fact that SNR is better not only at the surface but also at the center of the phantom, is a sign of the reduced coil noise contribution on the proposed array.

Besides the improved SNR, similar noise correlation values are also reported, despite the fact of avoiding coil overlapping. The 8-channel head coil array proved superior SENSE g-factor maps compared to a commercial 8-channel array, which demonstrate the more distinct coil profiles together with the higher SNR performance. This can be used in a hyperpolarization experiment to improve the temporal and/or spatial resolution of an acquisition through parallel imaging. Development of arrays with good parallel imaging performance is of particular interest for hyperpolarized ${ }^{13} \mathrm{C}$ imaging, due to the short half-life of the polarized agent in-vivo, and the theoretically non-existing SNR penalty [17].

The method described here to improve preamplifier 
decoupling and to minimize the coil noise contribution of the coil elements, is fully applicable to coil arrays of lower frequencies, for either imaging of other nuclei with lower Larmor frequency (e.g. ${ }^{15} \mathrm{~N}$ [27], [28]) or for ${ }^{1} \mathrm{H}$ imaging using low-field magnets. The latter option has attracted much attention lately, especially due to the advent of projects like the Open Source Imaging Initiative [29].

\section{CONCLUSION}

Mutual coupling can be a major source of SNR degradation in coil arrays where sample loading is low (low frequency and/or small elements). This is because of the lowered Q-factor of the individual coils, due to induced currents in the rest of the array. We show in this work that, with improved preamplifier decoupling, this can be avoided.

A method to improve preamplifier decoupling is presented, through the use of non-conventional matching impedances for the coils and preamps. In particular we show that matching the coils to a higher impedance than $50 \Omega$ is beneficial in terms of decoupling. It is also shown that, for a given real part of preamplifier impedance and matching impedance $Z_{0}$, a preamplifier with inductive input impedance provides higher decoupling than a traditional low (real) input impedance.

This method, using preamplifier with inductive input impedance, has been implemented in an 8-channel array for the human head for ${ }^{13} \mathrm{C}$ MRI at $3 \mathrm{~T}$ (32 MHz), with an almost 2-fold SNR enhancement when compared to a traditional array of similar size and number of elements. We demonstrate that the improved decoupling can be used to fabricate arrays without coil overlap in order to optimize the parallel imaging performance through better spatial encoding of the array.

\section{REFERENCES}

[1] J. H. Ardenkjaer-Larsen et al., "Increase in signal-to-noise ratio of \&gt; 10,000 times in liquid-state NMR," Proc. Natl. Acad. Sci., vol. 100 , no. 18 , pp. 10158-10163, Sep. 2003.

[2] K. . Golman et al., "Metabolic Imaging by Hyperpolarized 13C Magnetic Resonance Imaging for In vivo Tumor Diagnosis," Cancer Res., vol. 66, no. 22, pp. 10855-10860, Nov. 2006.

[3] C. H. Cunningham et al., "Hyperpolarized (13)C Metabolic MRI of the Human Heart: Initial Experience," Circ. Res., vol. 119, no. 11, pp. 1177-1182, Nov. 2016

[4] S. J. Nelson et al., "Metabolic Imaging of Patients with Prostate Cancer Using Hyperpolarized [1-13C]Pyruvate," Sci. Transl. Med., vol. 5, no. 198, pp. 198ra108-198ra108, Aug. 2013.

[5] D. H. Johansen et al., "Cryogenic Preamplifiers for Magnetic Resonance Imaging," IEEE Trans. Biomed. Circuits Syst., vol. 12, no. $1,2018$.

[6] C. Findeklee, "Array Noise Matching - Generalization, Proof and Analogy to Power Matching," IEEE Trans. Antennas Propag., vol. 59, no. 2, pp. 452-459, 2011.

[7] S. G. Hay, "Maximum-sensitivity matching of connected-array antennas subject to Lange noise constants," Int. J. Microw. Opt. Technol., vol. 5, no. 6, pp. 375-383, 2010.

[8] L. Belostotski et al., "Low-Noise Amplifier Design Considerations For Use in Antenna Arrays," IEEE Trans. Antennas Propag., vol. 63, no. 6, pp. 2508-2520, Jun. 2015.
J. A. de Zwart et al., "Design of a SENSE-optimized high-sensitivity MRI receive coil for brain imaging," Magn. Reson. Med., vol. 47, no. 6, pp. 1218-1227, Jun. 2002.

[10] C. von Morze et al., "An eight-channel, nonoverlapping phased array coil with capacitive decoupling for parallel MRI at 3 T," Concepts Magn. Reson. Part B Magn. Reson. Eng., vol. 31B, no. 1, pp. 37-43, Feb. 2007.

[11] N. De Zanche et al., "Modular design of receiver coil arrays," NMR Biomed., vol. 21, no. 6, pp. 644-654, Jul. 2008.

[12] P. B. Roemer et al., "The NMR Phased Array," MRM, vol. 225, pp. $192-225,1990$.

[13] A. Reykowski et al., "Design of Matching Networks for Low Noise Preamplifiers," MRM, no. 3, pp. 848-852, 1995.

[14] J. D. Sanchez-Heredia et al., "Low-Noise Active Decoupling Circuit and its Application to 13C Cryogenic RF Coils at 3T," TOMOGRAPHY, vol. 3, no. 1, p. 60:66, 2017.

[15] D. Kajfez and E. J. Hwan, "Q-Factor Measurement with Network Analyzer," IEEE Trans. Microw. Theory Tech., vol. 32, no. 7, pp. 666-670, Jul. 1984.

[16] D. Hoult, "The principle of reciprocity in signal strength calculations - A mathematical guide," Concepts Magn. Reson., vol. 12, no. 4, 2000

[17] R. F. Lee et al., "Advantages of parallel imaging in conjunction with hyperpolarized helium - A new approach to MRI of the lung," Magn. Reson. Med., vol. 55, no. 5, pp. 1132-1141, May 2006.

[18] A. Arunachalam et al., "Accelerated spectroscopic imaging of hyperpolarized C-13 pyruvate using SENSE parallel imaging," $N M R$ Biomed., no. June, pp. 867-873, 2009.

[19] A. Christ et al., "The Virtual Family - development of surface-based anatomical models of two adults and two children for dosimetric simulations," Phys. Med. Biol., vol. 55, no. 2, pp. N23-N38, Jan. 2010.

[20] K. P. Pruessmann et al., "SENSE : Sensitivity Encoding for Fast MRI," MRM, vol. 962, pp. 952-962, 1999.

[21] J. Mispelter et al., NMR Probeheads for Biophysical and Biomedical Experiments: Theoretical Principles \& Practical Guidelines. Imperial College Press, 2006.

[22] W. A. Edelstein, "Electronic decoupling of surface-coil receivers for NMR imaging and spectroscopy," J. Magn. Reson., vol. 67, no. 1, pp. $156-161,1986$.

[23] M. R. Bendall et al., "Elimination of Coupling between Cylindrical Transmit Coils and Surface-Receive Coils for in Vivo NMR M," $M R M$, vol. 163, pp. 157-163, 1986.

[24] I. E. Commission and others, "IEC 60601-2-33 Medical electrical equipment-Part 2-33: particular requirements for the safety of magnetic resonance equipment for medical diagnosis." Geneva: IEC, 2002 .

[25] J. Tropp et al., "Multi-channel metabolic imaging, with SENSE reconstruction, of hyperpolarized [1-13C] pyruvate in a live rat at 3.0 tesla on a clinical MR scanner," J. Magn. Reson., vol. 208, no. 1, pp. 171-177, Jan. 2011.

[26] M. Uecker et al., "ESPIRiT-an eigenvalue approach to autocalibrating parallel MRI: Where SENSE meets GRAPPA," Magn. Reson. Med., vol. 71, no. 3, pp. 990-1001, Mar. 2014.

[27] C. Cudalbu et al., "Feasibility of in vivo15N MRS detection of hyperpolarized $15 \mathrm{~N}$ labeled choline in rats," Phys. Chem. Chem. Phys., vol. 12, no. 22, p. 5818, 2010.

M. Durst et al., " $\alpha$-trideuteromethyl[15N]glutamine: A long-lived hyperpolarized perfusion marker," Magn. Reson. Med., vol. 76, no. 6, p. spcone-spcone, Dec. 2016.

[29] L. Winter et al., "Open Source Imaging Initiative," in ISMRM 2016 Annual Meeting \& Exhibition, 2016.

Copyright (c) 2018 IEEE. Personal use of this material is permitted. However, permission to use this material for any other purposes must be obtained from the IEEE by sending an email to pubs-permissions@ieee.org. 